\title{
Photonic crystal fiber modelling and applications
}

Bjarklev, Anders Overgaard; Broeng, Jes; Libori, Stig E. Barkou; Knudsen, Erik; Simonsen, Harald R.

\section{Published in:}

Proceedings of Optical Fiber Communication Conference and Exhibit, 2001

Link to article, DOI:

10.1109/OFC.2001.927299

Publication date:

2001

Document Version

Publisher's PDF, also known as Version of record

Link back to DTU Orbit

Citation (APA):

Bjarklev, A. O., Broeng, J., Libori, S. E. B., Knudsen, E., \& Simonsen, H. R. (2001). Photonic crystal fiber modelling and applications. In Proceedings of Optical Fiber Communication Conference and Exhibit, 2001 (Vol. 2) https://doi.org/10.1109/OFC.2001.927299

\section{General rights}

Copyright and moral rights for the publications made accessible in the public portal are retained by the authors and/or other copyright owners and it is a condition of accessing publications that users recognise and abide by the legal requirements associated with these rights.

- Users may download and print one copy of any publication from the public portal for the purpose of private study or research.

- You may not further distribute the material or use it for any profit-making activity or commercial gain

- You may freely distribute the URL identifying the publication in the public portal 


\title{
Photonic crystal fiber modelling and applications
}

\author{
A.Bjarklev, J.Broeng, S.E.Barkou Libori, E.Knudsen, and H.R.Simonsen* \\ Research Center COM, Technical University of Denmark,DTU, Building 345v, DK-2800 Kgs. Lyngby, Denmark \\ Phone: + 4545253808 , Fax: + 454593 6581, E-mail: ab@com.dtu.dk \\ *) Crystal Fibre A/S, Priorparken 878, DK-2605 Brondby, Denmark, Phone: + 45 4525 6364, Web address: www.crystal-fibre.com
}

\begin{abstract}
Photonic crystal fibers having a microstructured air-silica cross section offer new optical properties compared to conventional fibers for telecommunication, sensor, and other applications. Recent advances within research and development of these fibers will be presented. (c) 2000 Optical Society of America

OCIS codes: (060.0060) Fiber optics and optical communications; (060.2400) Fiber properties
\end{abstract}

\section{Introduction}

The concept of photonic crystals dates back to 1987 , when it was suggested [1,2] that the electronic bandgaps of semiconductors had an optical analogy in periodic dielectric structures. One of the most promising areas, where photonic crystals are finding use, is in optical fiber technology. This specific field of research is today about five years old [3,4], and it addresses the issue of periodically microstructured optical fibers with a high-index contrast (they typically consists of air holes in a silica background material). The fibers in question are often called Photonic Crystal Fibers (PCFs), and this new class of optical waveguides may conveniently be divided into two very different groups. The first is fibers having a high-index core (typically solid silica) surrounded by a two-dimensional photonic-crystal cladding-structure. These fibers have properties, which partly resemble those of conventional fibers due to the fact that the waveguidance is caused by a Total Internal Reflection (TIR) like principle; the higher refractive index of the core compared to the effective index of the photonic crystal cladding allows for traditional index guiding. It is, therefore, important to notice that these fibers, which we shall name TIR-PCFs, do in fact not rely on photonic bandgap (PBG) effects at all. Radically different to the TIR-PCFs are fibers, where the photoniccrystal-cladding structure is exhibiting PBG effect, and where this effect is utilised to confine light in the core region. These fibers, PBG-PCFs, show remarkable properties among which is the ability to confine and guide light along a core region having a refractive index below that of the cladding structure. While the TIR-PCFs were the first to be fabricated [5], truly PBG-guiding fibers were only recently experimentally demonstrated [6]. In this presentation, we shall address both types of fibers, and discuss the fundamental issues of modelling of photonic crystal fibers, with a specific focus on the most recent developments within the field.

\section{Modelling of photonic crystal fibers}

The theoretical treatment of photonic crystal fibers were initially dominated by the search for photonic bandgaps. This process was initially hindered by the lack of suitable theoretical models, since accurate modelling of photonic bandgap effects generally requires fully vectorial models, while scalar modelling of low-index contrast dielectric structures for the optical domain had been sufficient for most previous applications. Today, a wide range of theoretical models for general analysis of high-index contrast microstructures exist [7-10], and photonic crystals are being extensively exploited.

In order to qualitatively describe mode-propagation properties of the TIR- PCFs, the effective-index approach was proposed in 1997 [11]. The fundamental idea behind this work was first to evaluate the periodically repeated hole-in-silica structure of the cladding, and then (based on the approximate waveguiding properties of this cladding structure) replace the cladding by a properly chosen effective index. The core is pure silica, but the definition of the refractive index of the micro-structured cladding region is given in terms of the propagation constant of the lowestorder mode that.could propagate in the infinite cladding material. This scalar effective-index method has also been used as a basis for the approximate dispersion and bending analysis presented in [12]. It is noteworthy that the development of the technology towards larger air holes recently has made it relevant to approximate the fiber by an isolated strand of silica surrounded by air [17].

In 1990, the first method for finding PBGs in photonic crystals was described [7]. The method was closely related to methods used for calculating electronic bandgaps in semiconductor crystals, in that it described the magnetic field as a plane wave multiplied by a Bloch function with the two-dimensional periodicity of the photonic crystal. From Maxwell's equations an eigenvalue equation may now be formulated, which is well suited for calculating the PBGs of a periodic dielectric structure, since it describes the field and the structure as a Bloch function [13]. However, to include a core, one has to impose an artificial periodicity, which is handled numerically 
by creating a supercell with periodically repeated core-defects. This yields correct guided solutions, if the supercell is much larger than the guided mode-area [14]. Such a supercell approach requires a high number of plane waves, which initiated an interest in models capable of handling a large number of eigenvalues.

Based on the fact that silica is an isotropic material, we may choose to solve the waveguiding problem by the well-known vectorial transverse eigenvalue equation as outlined by Monro et al. [15]. In this formulation, the transverse wavevector $\mathrm{k}$ is scalar. Therefore, this formulation is suitable for finding guided solutions. Such formulations, therefore, avoid the supercell formulation of the core. One may then describe the cladding by a Fourier formulation (cosines), while the core-defect and the transverse electric field is described by localised functions [15]. The eigenvalue equation may then be recast into a matrix eigenvalue problem, where the matrix elements are found from overlap integrals, which may be calculated analytically.

\section{Basic operation of index guiding crystal fibers (TIR-PCFs)}

The first crystal fibers studied had a design similar to the one illustrated in Fig.1.a. The operation of such high-index core fibers may be understood from Fig. 1.b., which shows the effective index of the guided modes of a crystal fiber with relatively large air holes in the cladding structure. In addition, the figure shows the effective core and cladding indices and reveals that the high-index core crystal fibers have an operation that may be compared to traditional optical fibers operating by total internal reflection (TIR). This is seen from the fact that the effective indices of the guided modes are positioned between the core and cladding indices.
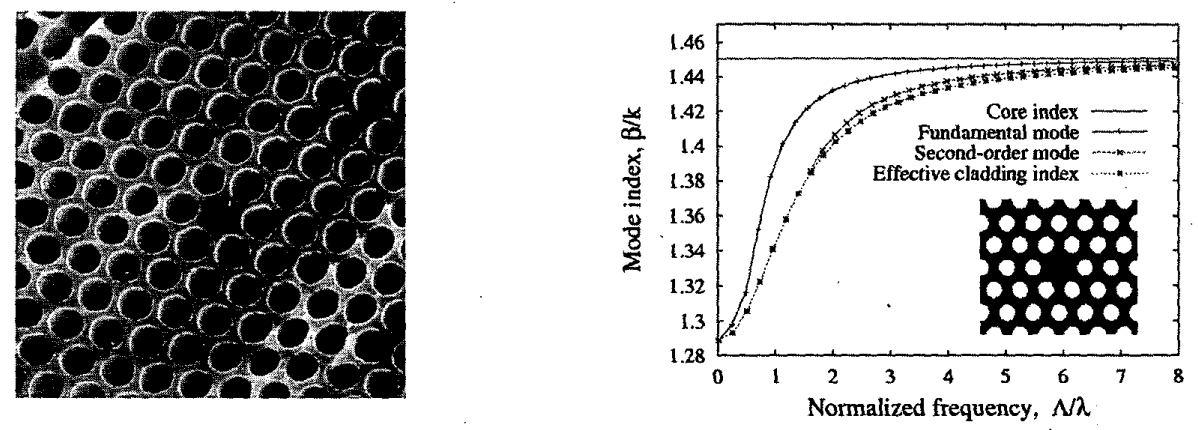

Fig 1.a. Scanning electron micrograph of inner part of an index guiding crystal fiber. The cladding of the fiber consists of a highly regular, triangular lattice of air holes. The core is formed by the omission of a single air hole. The photo is kindly borrowed from Crystal Fibre A/S (http://www.crystal-fibre.com)

Fig.1.b. Modal index illustration of the operation of a crystal fiber with a triangular air-hole lattice cladding structure and a high-index core formed from a single missing air hole. The figure is calculated [16] for normalized frequencies hence the properties (including the second-order mode cut-off) may be scaled to any wavelength range by scaling the centre-to-centre air hole spacing $\Lambda$ - provided that the refractive index of silica is equal to 1.45 at the given wavelength range. The fiber supports both a fundamental mode and a second-order mode with a normalized cut-off frequency, $\Lambda / \lambda$, around 1.5. The inset shows a cross-section of the PCF.

\section{Photonic bandgap guiding crystal fibers}

The principle on which the operation of the PBG guiding fibers relies is that by locally breaking the periodicity of a photonic crystal, a spatial region with optical properties different from the surrounding bulk photonic crystal can be created. If such a defect region supports modes with frequencies falling inside the PBG of the surrounding full periodic crystal, these modes will be strongly confined to the defect. A correctly designed defect may form a spatial region to which very strong transverse confinement is achieved. For this defect region to exhibit optical properties different from the surrounding periodic structure (i.e. be able to support a localized mode) it is not required that the defect region has a higher index than its surroundings - as in the case of index-guiding crystal fibers.

It has been found that silica-air photonic crystals with air holes arranged in a so-called honeycomb lattice are capable of exhibiting PBG effect for much smaller air holes than triangular (close-packed) photonic crystals [13]. Based on this, the first bandgap guiding crystal fibers (inset to Fig. 2) were designed. The inset shows how an extra air hole is introduced into the center of the fiber to act as a low-index defect region. To understand the waveguiding mechanism, it is valuable to consider the fiber using a modal-index illustration as used for the index-guiding crystal fiber. Fig. 2 shows such an illustration, and it is seen how two forbidden regions are opened up by photonic bandgap effects below the effective cladding index. No modes are seen to appear above the effective cladding index. However, the extra air hole causes a single mode to be confined to the core defect and, therefore, guided through the fiber in the frequency range for which the defect mode falls within the bandgap. Hence, the PBG fiber may only 
support guided modes within certain transmission windows. Depending on the extent of the PBGs, these transmission windows may be several microns wide and centered at near-infrared wavelengths [16].

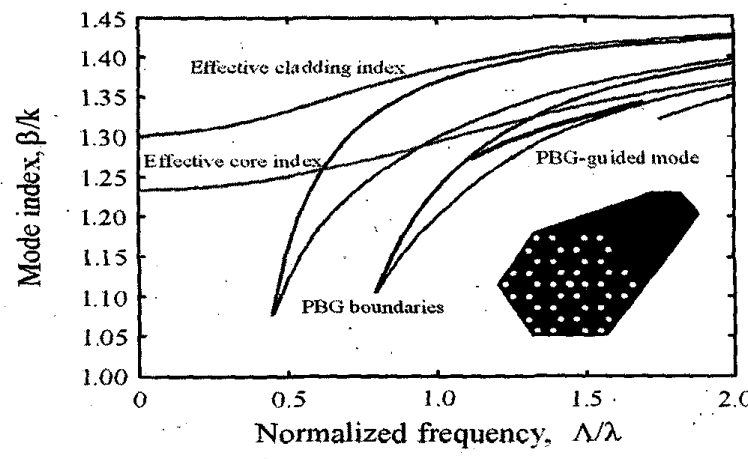

Fig.2 Illustration of the two lowest-frequency PBGs of a honeycomb crystal fiber with a cladding air filling fraction of $30 \%$ and a defect hole with same size as the cladding holes. Within the primary PBG a single degenerate mode is found. This defect mode may not propagate in the cladding structure (due to the photonic bandgap effect) and the mode is strongly localized to the region that breaks the periodicity of the photonic crystal, i.e., the region containing the

extra air hole forms the core of the PBG-fiber. The inset shows schematically the design of a honeycomb crystal fiber with the core region formed as a deliberately introduced spatial defect through the use 'of an extra air hole.

\section{Future perspectives}

An important point that must be addressed for the practical development of crystal fibers in the future is their tolerances towards minor fluctuation in the core-cladding region. Research also indicates that by deliberately enhancing asymmetries in crystal fibers, very strong birefringence may be achieved. Another potential application area of crystal fibers concerns the introduction of multiple cores, which are difficult to realize in conventional fibers. Multi-core crystal fibers may be used for various types of sensors (e.g., bend and strain sensors) or multi-channel telecommunication systems. Finally, yet another interesting aspect concerns utilizing the potential of photonic crystals for altering the properties of active materials. Although the alterations may not be as strong as for photonic crystals having a 3-D periodicity, the 2-D photonic crystal forming the fiber cladding may potentially provide a nonnegligible effect allowing improvement of fiber lasers and amplifiers.

\section{Conclusions}

Due to the unique ability of photonic crystal fibers to provide novel cut-off, spot-size, and dispersion properties, as well as allowing leakage-free waveguidance in a low-index core region, the potential future applications are numerous. We have emphasized some of the fundamental properties of these new fibers, pointed out their differences compared to conventional fibers and indicated some of their potential future applications.

\section{References}

[1] E.Yablonovitch, "Inhibited Spontaneous Emission in Solid-State Physics and Electronics", Physical Rev. Lett., 58, 2059-2062 (1987)

[2] S. John, "Strong Localization of Photons in Certain Disordered Dielectric Superlattices", Physical Rev. Lett., 58, 2486-2089 (1987).

[3] T.A.Birks, P.J.Roberts, P.St.J.Russell, D.M.Atkin, and T.J.Shepherd, "Full 2-D photonic bandgaps in silica/air structures", IEE Electr. Lett., 31, 1941-1943, (1995).

[4] OSA: J.C.Knight, T.A.Birks, D.M.Atkin, and P.St.J.Russell, "Pure silica single-mode fibre with hexagonal photonic crystal cladding", OFC'96 Optical Fiber Communication, Vol.2, Technical Digest Series, Paper CH35901 (1996).

[5] J.C. Knight, T.A. Birks, P.St.J. Russell, and D.M. Atkin., "All-silica single-modé optical fiber with photonic crystal cladding", Optics Letters, 21, 1547-1549 (1996).

[6] J.C. Knight, J. Broeng, T.A. Birks, P.St.J. Russell, "Photonic band gap guidance in optical fibers", Science, 282, 1476-1478 (1998).

[7] K.M.Ho, C.T.Chan, and C.M.Soukoulis, "Existence of a photonic gap in periodic dielectric structures", Phys. Rev. Lett., 65, 3152-3155 (1990).

[8] J.B. Pendry, A. MacKinnon; "Calculation of photon dispersion relations", Phys. Rev. Lett., 69, 2772-2775 (1992).

[9] A. Mekis, J.C. Chen, I. Kurland, S. Fan, P.R. Villeneuve, J.D. Joannopoulos, "High transmission through sharp bends in photonic crystal waveguides", Phys. Rev. Lett., 77, 3787-3790 (1996).

[10] G. Tayeb, D. Maystre, "Rigorous theoretical study of finite-size two-dimensional photonic crystals doped by microcavities", J. Opt. Soc. Am A, 14, 3323-3332 (1997):

[11] T.A.Birks, J.C.Knight, and P.St.J.Russell, "Endlessly single-mode photonic crystal fiber", Optics Letters, 22, $961-963$ (1997).

[12] A. Bjarklev, J. Broeng, S.E. Barkou, and K. Dridi.,." Dispersion properties of photonic crystal fibres", $24^{\text {th }}$ European Conference on Optical Communication, ECOC'98, Madrid, Vol.1, 135-136 (1998).

[13] J. Broeng, S.E. Barkou, A. Bjarklev, J.C. Knight, T.A. Birks, and P.St.J. Russell, "Highly increased photonic band gaps in silica/air structures", Optics Communications, 56, 240-244 (1998).

[14] R.D.Meade, A.M.Rappe. K.D.Brommer, J.D. Joannopoulos, and O.L.Alerhand, "Accurate theoretical analysis of photonic band-gap materials", Physical Review B, 48, 8434-8437 (1993).

[15] T.M.Monro, D.J.Richardson, N.G.R.Broderick, and P.J.Bennett, "Holey fibres: An efficient modal model", IEEE J. of Lightwave Techn., 17, 1093-1102 (1999)

[16] J.Broeng, T.Søndergaard, S.Barkou, P.Barbeito, and A.Bjarklev, "Waveguidance by the photonic bandgap effect in optical fibres", Pure and Applied Optics, 1, 477-482 (1999).

[17]. J.C.Knight, J.Arriaga, T.A.Birks, A.Ortigosa-Blanch, J.W.Wadsworth, and P.St.J.Russell, “Anomalous Dispersion in Phitinic Crystal Fiber", IEEE Photonics Techn. Lett., 12, 807-809 (2000). 